\title{
Influência da suplementação aguda e crônica de creatina sobre marcadores enzimáticos de dano muscular de ratos sedentários e exercitados com natação
}

CDD. 20.ed. 796.022

\section{Resumo}

0 objetivo deste estudo foi investigar os efeitos da suplementação aguda ( $5 \mathrm{~g} \cdot \mathrm{kg}^{-1}$ durante uma semana) e crônica (1 g. $\mathrm{kg}^{-1}$ durante quatro e oito semanas) de creatina $(\mathrm{Cr})$ sobre as concentrações plasmáticas de Creatina Quinase (CO), Lactato Desidrogenase (LDH) e Aspartato Aminotransferase (AST), de ratos sedentários e exercitados (natação a $80 \%$ da carga máxima tolerada). Setenta e dois ratos Wistar machos $(250 \pm 10 \mathrm{~g})$ foram utilizados e divididos igualmente em quatro grupos: ratos sedentários não suplementados (CON; $n=18)$; ratos exercitados não suplementados (NAT; $n=18)$; ratos sedentários e suplementados ( $C R E ; n=18)$; ratos exercitados e suplementados (CRE + NAT; $n=18$ ). Ao final da primeira, quarta e oitava semanas, seis animais de cada grupo foram sacrificados. Os resultados demonstraram: 1) ao final da primeira semana elevação plasmática de CQ, LDH e AST nos grupos NAT-1 e CRE+NAT-1 em relação aos grupos CON-1 e CRE-1; 2) ao final da quarta semana valores superiores para CQ e LDH somente no grupo NAT-4; e 3 ) ao final da oitava semana, somente os valores de AST do grupo CRE-8 diferiram dos demais. Estes achados sugerem que a suplementação de $\mathrm{Cr}$ : 1) não afeta o dano muscular em ratos submetidos a uma semana de treinamento físico de alta intensidade em meio aquático; 2) pode ser capaz de reduzir o dano muscular após quatro semanas de treinamento; e 3) após oito semanas de suplementação de $\mathrm{Cr}$, o dano muscular parece ser atenuado pelo próprio exercício, anulando os efeito da $\mathrm{Cr}$.

UnItermos: Recurso ergogênico; Dano muscular; Estresse oxidativo.

\section{Introdução}

Nas últimas décadas, a lesão muscular exercícioinduzida tem sido amplamente estudada (CHEUNG, Hume \& Maxwell, 2003; Coudreuse, Dupont \& Nicol, 2004; Endoh, NaKajima, SaKamoto \& Komiyama, 2005; Lieber \& Friden, 2002). Tem sido relatado o aumento da permeabilidade da sarcolema com consequente liberação de enzimas musculares, quando da realização de exercícios físicos desabituais (Brancaccio, Maffulli \& Limongelli, 2007). Nesse sentido, Neubauer, Konig e Wagner (2008) e Margaritis, Tessier, Verdera, Bermon e Marconet (1999) consideram que as enzimas Creatina Quinase (CQ), Lactato desidrogenase (LDH) e Aspartato Aminotransferase (AST) podem ser consideradas marcadores bioquímicos indiretos do dano muscular sendo comumente usadas na prática clínica. Em função da lesão muscular exercício-induzida, o desempenho físico é prejudicado, e dessa forma, justifica-se a investigação de estratégias medicamentosas e nutricionais quanto à possibilidade de interferirem positivamente sobre esse aspecto (BEMBEN \& LAMONT, 2005). 
De acordo com esse contexto, a suplementação de creatina $(\mathrm{Cr})$ tem sido investigada quanto ao seu possível benefício sobre o tecido muscular estriado esquelético submetido ao estresse promovido pelo exercício físico. Bassit, CuRI e Costa Rosa (2008) e Santos, Bassit, Caperuto e Costa Rosa (2004) concluíram que a suplementação de $\mathrm{Cr}$ reduziu o dano muscular e a resposta inflamatória através da observação de que a $\mathrm{Cr}$ atenua o incremento pós-exercício de $\mathrm{CQ}$, prostaglandina $\mathrm{E} 2$, fator de necrose tumoral $\alpha \mathrm{e}$ LDH em maratonistas após $30 \mathrm{~km}$ de corrida. Recentemente Bassit, Pinheiro, Vitzel, Sproesser, Silveira e CURI (2010) observaram que a suplementação de $\mathrm{Cr}$ exerceu um efeito protetor sobre a lesão muscular induzida por atividades contráteis extenuantes tanto em humanos como em ratos. Contudo, ao contrário desses resultados, RawsOn, GunN e ClaRKSON (2001) verificaram que após o treinamento muscular, embora houvesse lesão de natureza mecânica com consequente redução da força e aumento da concentração sérica protéica, não foram encontradas diferenças entre os atletas que recebiam placebo ou suplementação de $\mathrm{Cr}$. Em modelo animal, WarRen, FenNessy e MillardSTAFFORD (2000) também não encontraram melhora na perda de força muscular associada à suplementação de Cr após o dano muscular exercício-induzido.

Embora a literatura relacione o possível efeito protetor da suplementação de $\mathrm{Cr}$ em atividades exaustivas como a maratona (BASSIT, CuRI \& COSTA Rosa, 2008; Bassit et al., 2010; SANTOS, BASSIT, CAPERUTO \& Costa RosA, 2004) faltam estudos que esclareçam a resposta de marcadores indiretos de lesão muscular em sessões de exercícios exaustivos de outras modalidades esportivas, como a natação. Sendo assim, o objetivo deste estudo foi investigar os efeitos da suplementação de $\mathrm{Cr}$ sobre marcadores enzimáticos de dano muscular em ratos sedentários e exercitados com natação, tanto em caráter agudo, como crônico.

\section{Métodos}

\section{Amostra}

Foram utilizados 72 ratos $(250 \pm 10 \mathrm{~g})$ machos Wistar ("Rattus Norvegicus"), adultos jovens (10-12 semanas de idade), obtidos da fazenda Bem-te-vi (Paulínia/SP). Os animais foram mantidos individualmente em caixas de polietileno no biotério do Laboratório de Fisiologia e Farmacodinâmica do Instituto de Pesquisa e Desenvolvimento da Universidade do Vale do Paraíba com temperatura (22-25 ${ }^{\circ} \mathrm{C}$ ), umidade relativa (40-60\%) e fotoperíodo (ciclo de 12 horas claro-escuro) controlados. Além disto, todos os animais tiveram acesso à ração peletizada e água "ad libitum". O estudo teve duração de oito semanas, os animais foram divididos igualmente em quatro grupos experimentais: ratos sedentários não suplementados (CON; $\mathrm{n}=18$ ); ratos exercitados não suplementados (NAT; $\mathrm{n}=18$ ); ratos sedentários e suplementados (CRE; $\mathrm{n}=18$ ); ratos exercitados e suplementados (CRE+NAT; $\mathrm{n}=18)$. Todos os procedimentos adotados neste estudo estavam de acordo com os princípios de manuseio e cuidado com animais de laboratório preconizados pelo COBEA (Colégio Brasileiro de Experimentação Animal) e aprovado pelo Comitê de Ética e Pesquisa da UNIVAP (Protocolo no L022-2005-CEP).

\section{Procedimentos}

Todos os animais foram submetidos a um período de adaptação à natação (30 minutos diários sem carga, durante cinco dias consecutivos) para reduzir fatores ligados ao estresse promovido pela atividade do nado (Voltarelli, Gobatto \& Mello, 2002). Durante este período a creatina não foi administrada. Após a adaptação, os animais foram individualmente submetidos ao teste de carga máxima (TCM) (Osorio, Christofani, D’Almeida, Russo \& Picarro, 2003a; Osorio, Sillveira, Maldjian, Morales, Christofani \& Russo 2003b). O TCM é caracterizado pela introdução de células de carga (placas de cobre) correspondendo a $0 \%, 1 \%, 2 \%$, $3 \%$, etc. da massa corporal total do animal, colocadas a cada três minutos, em uma tira elástica posicionada junto ao tórax do animal até sua exaustão. A exaustão foi determinada pela incapacidade do animal manterse sob a superfície da água por oito segundos (OsORIO et al., 2003a, 2003b). Quando da exaustão, o TCM encerrava-se e as placas de cobre encontradas na tira elástica do animal eram pesadas para verificação da carga máxima tolerada. Esse procedimento permitiu o ajuste da carga de trabalho para o treinamento físico a $80 \%$ da carga máxima. 
O treinamento físico a $80 \%$ da carga máxima foi realizado em grupos de seis animais devido à promoção de exercício mais vigoroso quando comparado ao nado individual (Voltarelli, Gobatto \& Mello, 2002). Foi demonstrado previamente que essa intensidade de exercício promove significativo aumento de lactato sanguíneo indicando importante estresse físico (SoUZA, SANTOS, Osorio, Cogo, Prianti Júnior, Martins \& Ribeiro, 2006). Este treinamento ocorreu cinco vezes por semana com sessôes diárias de treinamento de 30 minutos somente nos grupos experimentais NAT e CRE+NAT. Foram utilizados coletes contendo pesos de chumbo e posicionados junto ao tórax de cada animal (Osorio et al., 2003a, 2003b; VoltarelLi, Gobatto \& Mello, 2002). Ao final de cada semana experimental, novo TCM foi realizado para possíveis reajustes da carga de treinamento. $\mathrm{O}$ protocolo de natação foi realizado em um tanque de amianto com capacidade para 250 litros de água, mantidos a temperatura de $35 \pm 2{ }^{\circ} \mathrm{C}$ (Osorio et al., 2003b).

A suplementação foi realizada por meio de uma sonda oro-esofágica (1 $\mathrm{mm}$ de diâmetro; $3 \mathrm{~cm}$ de comprimento) adaptada a uma seringa de $3 \mathrm{ml}$, tendo a água como veículo de infusão ("gavage"). Este procedimento ocorreu diariamente após o período de adaptação ao nado duas horas antes do treinamento físico. Foi instituída durante a primeira semana do experimento ("loading phase") a dose diária de $5 \mathrm{~g}$ de $\mathrm{Cr} / \mathrm{kg}$ de massa corporal do animal e, após a primeira semana (fase de manutenção), a dose diária de $1 \mathrm{~g}$ de $\mathrm{Cr} / \mathrm{kg}$ de massa corporal do animal para todos os animais suplementados (CRE e CRE+NAT). Considerando que uma dose diária de $300 \mathrm{mg}$ de $\mathrm{Cr} / \mathrm{kg}$ de massa corporal é rotineiramente usada em outros estudos com roedores (BRANNON, Adams, Gonniff \& Baldwin, 1997; Gagnon, Maguire, Macdermott \& Bradford, 2002; Young \& Young, 2007) e equivale ao regime de dosagem utilizado por humanos para promover efeitos ergogênicos associados com aumento do conteúdo intramuscular de Cr (BRANNON et al., 1997; Tarnopolsky, Bourgeois, Snow, Keys, Roy, Kwieeien \& Turnbull, 2003), o protocolo de suplementação utilizado no presente estudo deve ser considerado suprafisiológico. Os animais dos grupos CON e NAT também foram submetidos ao estresse da "gavage", contudo, sem a suplementação de Cr. Para facilitar a absorção do suplemento e minimizar qualquer risco de contaminação do produto foi utilizada creatina micronizada com pureza assegurada.

Os quatro grupos experimentais (CON, NAT, CRE e CRE+NAT) foram constituídos inicialmente de 18 animais cada um. Contudo, o modelo experimental exigiu a subdivisão dos grupos experimentais em três subgrupos (cada um com seis animais), e de acordo com o período do estudo analisado (após uma, quatro e oito semanas). Após o período de estudo instituído para cada grupo experimental, os animais dos respectivos grupos foram sacrificados. O sacrifício foi realizado após dois dias da última sessão de treinamento, para atenuar os efeitos agudos do exercício (NAKAO, OOKAWARA, Kizaki, Oh-Ishi, Miyazaki, Haga, Sato, Ji \& Ohno, 2000). Foi adotado como anestésico o etoxietano, pois promove ação depressora no Sistema Nervoso Central sem provocar qualquer disfunção tecidual. Os animais foram sacrificados através de administração de Cloreto de Potássio (KCl) 20\% intracardíaco, ainda sob efeito anestésico, e após a realização dos procedimentos experimentais listados a seguir.

Alcançada a indução anestésica, as amostras sanguíneas $(3 \mathrm{ml})$ foram coletadas da veia cava inferior dos animais com seringas de $5 \mathrm{ml}$, utilizando agulhas de calibre $25 \times 7 \mathrm{~mm}$, após afastamento prévio das vísceras anteriores ao vaso. O sangue coletado foi colocado em tubos de ensaio sem a presença de anticoagulantes e centrifugado a $3000 \mathrm{rpm}$ por 15 minutos a $4^{\circ} \mathrm{C} \mathrm{em}$ centrífuga (Excelsa II ${ }^{\circledR}$ - Mod. 206BL). Em seguida, separadas as amostras plasmáticas do sangue de cada animal, essas foram conduzidas para as determinaçōes bioquímicas específicas através de kits comerciais (Laborlab®), por meio de um espectrofotômetro (Hitachi® - Modelo U-2001). Foram realizados os testes de determinação plasmática das enzimas Creatina Quinase (CQ), Lactato Desidrogenase (LDH) e Aspartato Aminotransferase (AST).

\section{Análise estatística}

Os resultados foram expressos como média \pm desvio padrão. Foi utilizada análise de variância (ANOVA) 4 x 4 para medidas repetidas entre os grupos experimentais nos diferentes períodos experimentais. $\mathrm{O}$ teste "post hoc" de Tukey, para comparaçôes múltiplas, foi empregado para a identificação das diferenças específicas nas variáveis em que os valores de $\mathrm{F}$ encontrados foram superiores ao critério de significância estatística estabelecido $(\mathrm{p}<0,05)$. 


\section{Resultados}

As FIGURAS 1, 2 e 3 revelam as concentrações plasmáticas de Creatina Quinase (CQ), Lactato Desidrogenase (LDH) e Aspartato Aminotransferase (AST), respectivamente. Após a primeira semana de estudo, observamos um aumento estatisticamente significativo $(\mathrm{p}<0,05)$ de CQ, LDH e AST quando comparamos os grupos NAT-1 e CRE+NAT-1 "versus" os grupos CON-1 e CRE-1. Ao final da quarta semana experimental observamos que os animais do grupo NAT-4 apresentaram valor médio

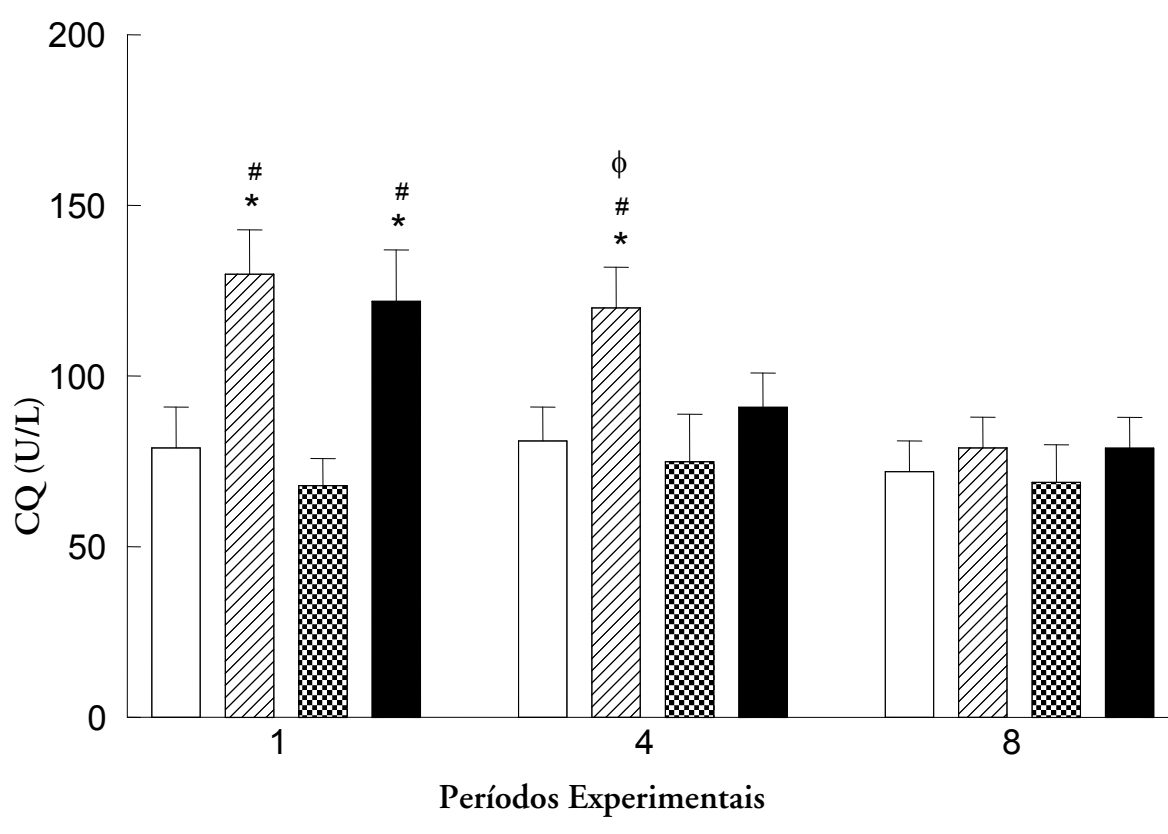

de CQ e LDH superior ( $p<0,05)$ em relação os demais grupos (CON-4, CRE-4 e CRE+NAT-4). Com relação à análise plasmática de AST ao final da quarta semana, não foram encontradas diferenças significativas $(p>0,05)$ entre os grupos experimentais. No último período experimental, com exceção dos achados para AST no grupo CRE-8 em relação os demais grupos (CON-8, CRE-8 e CRE+NAT-8), não encontramos diferenças significativas de CQ e $\mathrm{LDH}$ entre os grupos experimentais.
Resultados expressos como média \pm desvio padrão.

$\mathrm{CON}=$ grupos controle; NAT = grupos natação, CRE = grupos creatina; $\mathrm{CRE}+\mathrm{NAT}=$ grupos creatina natação. * $p<0,05$ "versus" CON. $\# p<0,05$ "versus" CRE; $\Phi$ "versus" CRE+ NAT.

Resultados expressos como média \pm desvio padrão.

$\mathrm{CON}=$ grupos controle; NAT = grupos natação; CRE = grupos creatina; $\mathrm{CRE}+\mathrm{NAT}=$ grupos creatina natação. * $p<0,05$ "versus" CON; \# $p<0,05$ "versus" CRE; $\Phi$ "versus" CRE+ NAT.
FIGURA 1 - Creatina Quinase (CQ) plasmática.

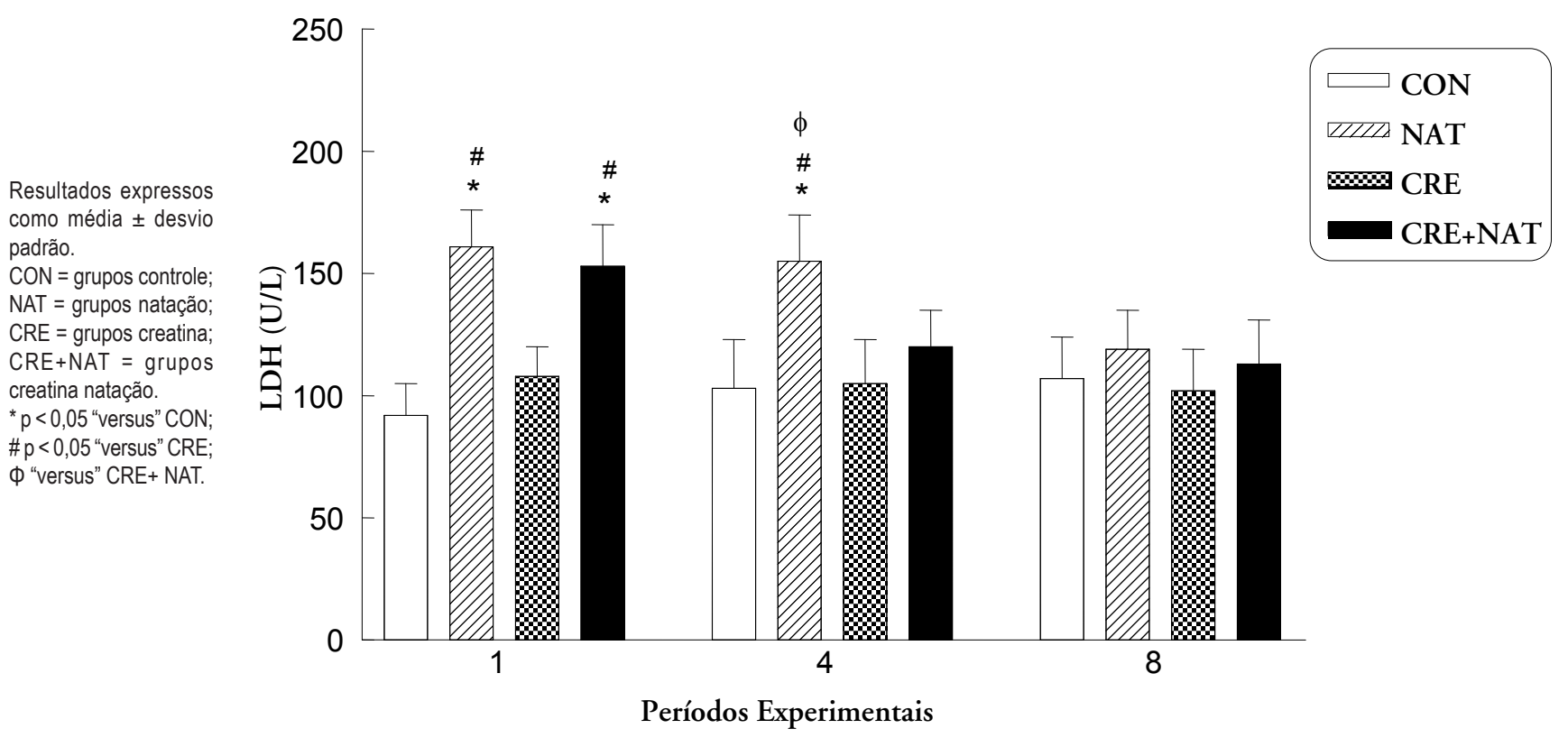

FIGURA 2 - Lactato Desidrogenase (LDH) plasmático.

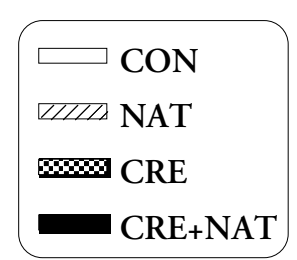

CRE+NAT 


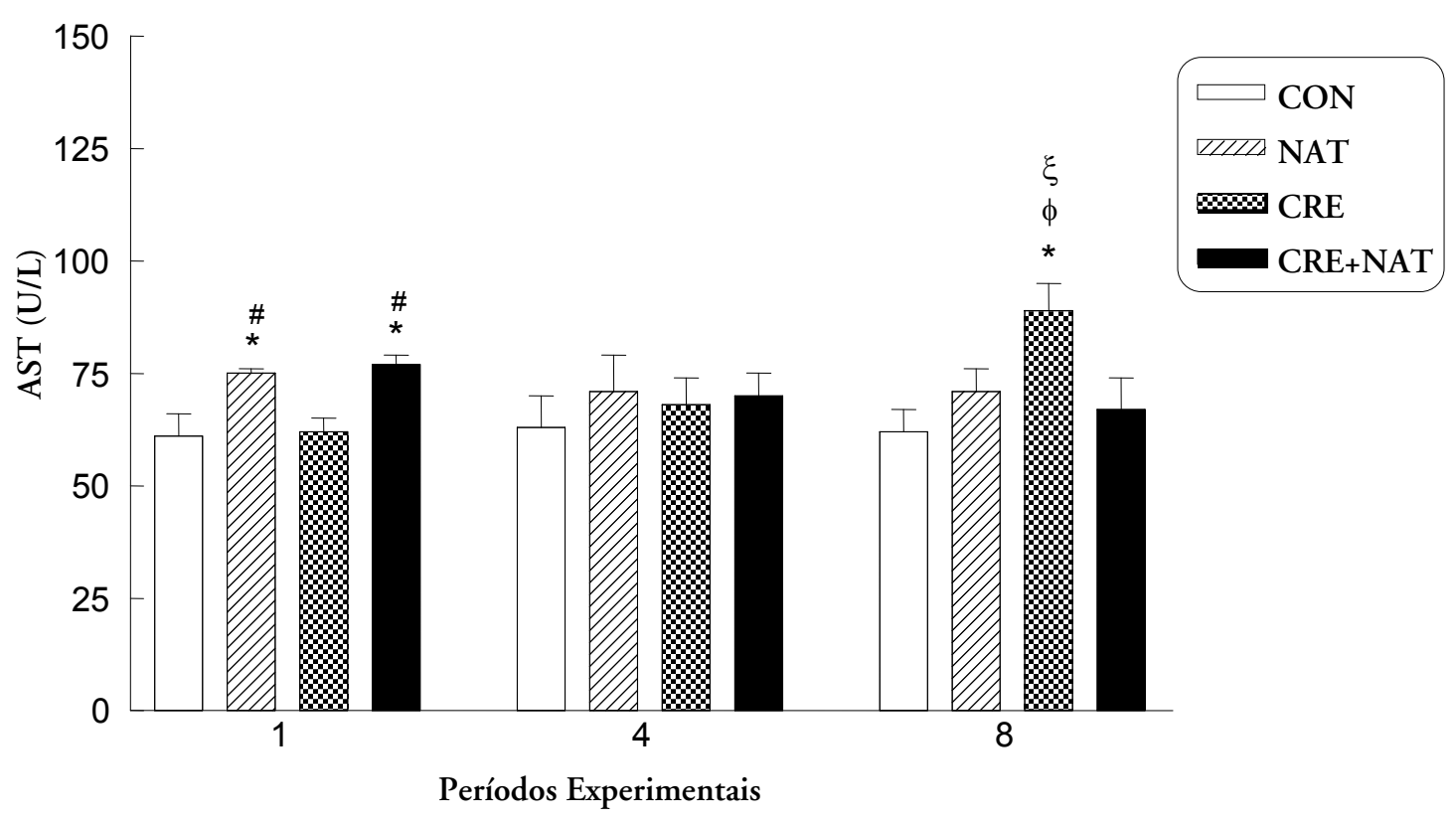

FIGURA 3 -Aspartato Aminotransferase (AST) plasmático.

\section{Discussão}

A suplementação de Cr tem sido amplamente investigada com propósitos ergogênicos e terapêuticos (Bemben \& Lamont, 2005; Bender, Auer, Merl, Reilmann, Saemann, Yassouridis, Bender, Weindl, Dose, Gasser \& Klopstock, 2005; Gualano, Artioli, Poortmans \& Lancha Junior, 2010). Diversos mecanismos foram propostos para justificar seus aspectos ergogênicos: aumento da disponibilidade e resíntese de PCr, aumento do tamponamento de íons hidrogênio $(\mathrm{H}+)$ reduzindo a acidez muscular, redução do acúmulo de lactato, aumento da atividade da enzima Citrato Sintase (CS), um marcador da capacidade oxidativa e aumento da massa muscular (BRANNON et al., 1997; SouZA et al., 2006). Terapeuticamente, tem sido descrito três possíveis mecanismos de prevenção ou redução do dano celular associados com a suplementação de $\mathrm{Cr}$ : estabilização de membranas celulares, homeostase energética mitocondrial e redução de lesões oxidativas (Persky \& Brazeau, 2001; Saks \& Strumia, 1993). Nesse sentido, o presente estudo objetivou investigar os efeitos da suplementação de Cr sobre marcadores enzimáticos de dano muscular em modelo animal, tanto em caráter agudo, como crônico.

É estabelecido que o exercício físico de longa duração e intensidade pode gerar dano muscular induzido por hipóxia (estresse oxidativo) com consequente elevação plasmática das enzimas AST, LDH e CQ
(Cheung, Hume \& Maxwell, 2003; Coudreuse, Dupont \& Nicol, 2004; ENDOH et al., 2005; LiEBER \& FrIDEN, 2002). No presente estudo, após a primeira semana foi observado significativo aumento das enzimas analisadas nos grupos que praticavam a natação (NAT-1 e CRE+NAT-1). A natação per si representa um importante agente causador de estresse quando imposta a ratos (Voltarelli, GoBATTO \& MELlo, 2002). Considerando a associação desse fato com o protocolo de treinamento de alta intensidade adotado ( $80 \%$ da carga máxima, durante 30 minutos diários), fica justificado porque após a primeira semana experimental, somente nos grupos exercitados as enzimas CQ, LDH e AST estavam significativamente aumentadas. Ou seja, é provável que o estresse promovido pela própria atividade física tenha sido o responsável por esse fenômeno.

Conforme descrito por Komulainen, Takala e ViнкO (1995), a atividade sérica da enzima CQ pode aumentar sem concomitantemente lesão muscular após um protocolo de natação e que o dano muscular pode ocorrer sem um aumento significativo desse mesmo marcador após um protocolo em esteira utilizando ratos como modelo animal. Por outro lado, Chen, Serfass e Apple (2000) concluíram que a natação prolongada e de forma intensa acarreta maior perda das frações de CQ muscular e mitocondrial tanto em fibras musculares vermelhas quanto
Resultados expressos como média \pm desvio padrão.

$\mathrm{CON}=$ grupos controle; NAT = grupos natação; $\mathrm{CRE}$ = grupos creatina; $\mathrm{CRE}+\mathrm{NAT}=$ grupos creatina natação. * $p<0,05$ "versus" CON; \# $p<0,05$ "versus" CRE; $\Phi$ "versus" CRE+ NAT; $\xi$ "versus" NAT. 
brancas. Nesse sentido, fica claro a variabilidade na medida dessa enzima CQ, não permitindo que este marcador indireto de dano muscular, seja o mais representativo para analisar esse fenômeno. Contudo, é importante ressaltar que no presente estudo outras duas enzimas de ocorrência preferencial no tecido muscular estriado esquelético (AST e LDH) também estavam aumentadas após a primeira semana nos grupos exercitados, reforçando a provável lesão muscular induzida pela natação.

Ao observar que o grupo CRE+NAT-1 apresentou resultados semelhantes aos encontrados pelo grupo NAT-1 é possível descartar um possível efeito agudo protetor da suplementação da $\mathrm{Cr}$ sobre o tecido muscular estriado esquelético submetido ao estresse do nado. Embora Rawson, Conti e Miles (2007) e Rawson, Gunn e ClarKSON (2001) tenham utilizado um modelo de lesão muscular diferente do presente estudo (exercícios excêntricos), esses autores ao analisarem as concentrações séricas de enzimas musculares também não observaram redução do dano muscular após a suplementação aguda de $\mathrm{Cr}$ ( 5 dias de suplementação).

Por outro lado, alguns estudos têm demonstrado um potencial efeito da suplementação de $\mathrm{Cr}$ sobre a recuperação e manutenção da integridade muscular tanto em modelos experimentais de miopatias quanto após atividades contráteis extenuantes (BASSIT, CURI, \& Costa Rosa 2008; Bassit et al., 2010; Menezes, Sobreira, Neder, Rodrigues-Júnior \& Martinez 2007; SANTOS et al., 2004). No presente estudo foi observado que após a quarta semana experimental somente o grupo NAT-4 continuou apresentando valores enzimáticos significativamente superiores de LDH e CQ. É importante destacar que os animais do grupo CRE+NAT-4 apresentaram redução nos valores plasmáticos dos marcadores indiretos do dano muscular, sugerindo interferência da suplementação de $\mathrm{Cr}$ sobre esse fenômeno. É provável que o protocolo de natação instituído neste estudo durante quatro semanas tenha sido suficientemente intenso para gerar lesão muscular associada ao estresse oxidativo (SouZA et al., 2006).

A homeostase energética mitocondrial éfundamental para impedir a ativação da degradação de purinas para fornecer adenosina monofosfato (AMP) através do ciclo de Lowenstein. As principais consequências da ativação dessa via são a produção paralela de amônia, hipoxantina, xantina, urato e de espécies reativas de oxigênio (EROs) (Halliwell, 2006). Bellinger, Bold, Wirson, Noakes e MyBURGH (2000) demonstraram que a suplementação de $\mathrm{Cr}$ previamente ao exercício intenso resultou em queda, tanto na produção de amônia como na de hipoxantina. Esses autores hipotetizaram que o aumento nos estoques intracelulares de Cr pelo treinamento físico, ou seu consumo adicional previamente à realização do exercício intenso, possa servir como antioxidante indireto. Wyss e SCHULZE (2002) sugeriram que a suplementação de $\mathrm{Cr}$ interfere com a homeostase de cálcio intracelular favorecendo mais um possível mecanismo antioxidante indireto desse suplemento. Além disso, a suplementação de $\mathrm{Cr}$ parece diminuir os níveis de homocisteína (aminoácido promotor de formação de EROs, especialmente o ânion superóxido $\mathrm{O}_{2}^{-}$), justificando novamente seu efeito antioxidante indireto (Deminice, Villhena, Portari \& Jordão, 2007).

Recentemente, autores têm sugerido e testado o potencial da $\mathrm{Cr}$ em agir diretamente na remoção de EROs (Sestili, Martinelli, Bravi, Piccoli, Curci, Battistelli, Falcieri, Agostini, Gioacchini \& STOCСнi, 2006). Esses autores observaram que a suplementação de $\mathrm{Cr}$ atenuou os efeitos citotóxicos causados por oxidantes em todas as células em cultura estudadas (promonócitos, células endoteliais e mioblastos. Além disso, Dangott, Schultz e MOZDZIAK (2000) observaram que a suplementação de $\mathrm{Cr}$ incrementou a ativação de células satélites musculares, as quais contribuem com o processo de regeneração do tecido muscular esquelético.

Todos esses aspectos supracitados, embora não tenham sido avaliados diretamente neste estudo, poderiam justificar porque os valores plasmáticos das enzimas CQ e LDH apresentaram reduzidos no grupo CRE+NAT-4 quando comparados ao grupo NAT-4. Ainda ao final desse período experimental, a enzima AST apresentou-se de forma semelhante entre os grupos exercitados. Para GuYOTTO e Alves (2001) esta resposta da AST é característica de dano agudo com evolução para o restabelecimento de integridade tecidual. Na tentativa de melhor explicar esse achado também hipotetizou-se que o alto peso molecular dessa enzima comparado as enzimas CQ e LDH reduz sua capacidade de difusão de membrana dificultando sua elevação plasmática (COOKE, RYBALKA, WiLliams, Cribb \& Hayes, 2009); e ao final da quarta semana de treinamento é possível algum grau de adaptação ao nado que decline a intensidade da lesão muscular (Brancaccio, Maffulli \& Limongelli, 2007).

Tem sido descrito na literatura que o treinamento físico é capaz de reduzir o dano muscular (LAVENDER \& Nosaka, 2008; Nosaka, Newton, Sacco, Chapman $\&$ LAVENDER, 2005). Embora este fenômeno esteja intimamente ligado às açõos excêntricas, e o modelo utilizado neste estudo não priorize atividade muscular excêntrica de alta intensidade, tal fato não pode ser completamente descartado. Esse aspecto pode ser melhor observado nos 
resultados obtidos após a oitava semana experimental. Ambos os grupos exercitados (CRE+NAT-8 e NAT-8) apresentaram valores semelhantes $(\mathrm{p}>0,05)$ em relação os grupos sedentários, justificando o efeito protetor da carga de treinamento ao longo do tempo (BRANCACCIO, MafFulli \& Limongelli, 2007).

Pode-se especular que a ausência de alteração das enzimas na semana 8 deva-se ao efeito crônico do exercício sobre o dano muscular, ocultando os efeitos da Cr sobre essa variável e, dessa forma, tornando-a desnecessária (para esse fim) após períodos mais longos de treinamento, nos quais sabidamente o dano tende a diminuir.

De forma interessante, ao final da oitava semana, a concentração plasmática de AST foi significativamente superior no grupo CRE-8 em relação aos demais grupos experimentais. Acreditamos que como essa enzima está associada a distúrbios de outros órgãos como fígado, esse achado não esteja diretamente relacionado com uma resposta muscular. De fato, a análise histológica do fígado dos animais desse grupo revelou indícios de lesão hepática, remetendo-nos a hipotetizar que a suplementação de $\mathrm{Cr}$ em animais sedentários em longo prazo pode causar algum dano hepático (SouzA, Miranda, Xavier, Osorio, Gouvea, Cogo, Vieira \& Ribeiro, 2009). É importante ressaltar que a dose utilizada é suprafisiológica e estudos com humanos refutam repetitivamente dano hepático induzido pela suplementação de Cr (Kreider, Melton, Rasmussen, Greenwood, Lancaster, Cantler, Milnor \& Almada, 2003; MaYhew, MaYhew \& Ware 2002;
Terjung, Clarkson, Eichner, Greenhaff, Hespel, Israel, Kraemer, Meyer, Spriet, Tarnopolsky, WagenMaKers \& Williams, 2000). Além disso, existem grandes diferenças no que diz respeito ao metabolismo da $\mathrm{Cr}$ (p.ex: biodisponibilidade, captação tecidual) entre espécies (TARNOPOLSKY et al., 2003).

Por fim, é necessário salientar que este estudo trata-se de um ensaio experimental aberto (não cego); a suplementação de $\mathrm{Cr}$ produz efeitos e respostas distintas (biodisponibilidade, captação, efeitos ergogênicos e adversos) mesmo em espécies evolutivamente bem próximas, o que não permite o extrapolamento pleno dos resultados obtidos para estudos envolvendo seres humanos; os marcadores de lesão muscular utilizados são enzimas com alta variabilidade plasmática sendo necessário estudos adicionais que façam a aferição de outros marcadores mais sensíveis da lesão muscular; é evidente a necessidade de monitorar mais precisamente o estresse físico, como por exemplo através da verificação do consumo de oxigênio e monitoramento de EROs. Contudo, de forma geral, ao considerarmos a metodologia empregada, os resultados desse estudo nos permite sugerir que a suplementação de $\mathrm{Cr}$ não afeta o dano muscular em ratos submetidos a uma semana de treinamento físico de alta intensidade em meio aquático. Os achados indicam, no entanto, que a suplementação de $\mathrm{Cr}$ pode ser capaz de reduzir o dano muscular após quatro semanas de treinamento. Finalmente, após oito semanas de suplementação, o dano muscular parece ser atenuado pelo próprio exercício, anulando os efeito da Cr.

\section{Abstract}

Influence of short and long term creatine supplementation on enzymatic markers of muscle damage in sedentaries and exercised rats

The aim of this study was to investigate the effects of the short-term ( $5 \mathrm{~g} \cdot \mathrm{kg}^{-1}$ to 1 week) and long-term (1 g. $\mathrm{kg}^{-1}$ to 4-8 weeks) creatine supplementation ( $\mathrm{Cr}$ ) on the Creatine Kinase (CK), Lactate Dehydrogenaze (LDH) and Aspartate Aminotransferase (AST) plasmatic concentrations of sedentary and exercised (swimming to $80 \%$ of the tolerated maximum load) rats. Seventy two Wistar males rats $(250 \pm 10 \mathrm{~g})$ were equally divided in four groups: sedentary rats without supplementation (CON; $n=18)$; exercised rats without supplementation (NAT; $n=18$ ); sedentary rats with supplementation (CRE; $n=18$ ); exercised rats with supplementation (CRE+NAT; $n=18)$. At the end of the first, fourth and eighth weeks six animals of each group were sacrificed. The results demonstrated: 1 ) improvement of CK, LDH and AST in NAT- 1 and CRE+NAT-1 groups vs. CON-1 and CRE-1 groups after the first experimental week; 2 ) higher values of CK and LDH only NAT-4 group after four experimental weeks; and 3) only AST from CRE-8 group differed from other groups at the end of the eighth experimental week. These findings suggest that the Cr supplementation: 1) does not affect the muscle damage on swimming exercised rats after 
one week of high intensity training; 2) could be able to reduce the muscle damage after 4 weeks of training; and 3) after eight weeks of $\mathrm{Cr}$ supplementation, the muscle damage seems to be attenuated by the exercise, nullifying the effects of $\mathrm{Cr}$.

UnITERMS: Ergogenic resource; Muscular damage; Oxidative stress.

\section{Referências}

BASSIT, R.A.; CURI, R.; COSTA ROSA, L.F. Creatine supplementation reduces plasma levels of pro-inflammatory cytokines and PGE2 after a half-ironman competition. Amino Acids, Wien, v.35, n.2, p.425-31, 2008.

BASSIT, R.A.; PINHEIRO, C.H.J.; VITZEL, K.F.; SPROESSER, A.J.; SILVEIRA, L.R.; CURI, R. Effect of short-term creatine supplementation on markers of skeletal muscle damage after strenuous contractile activity. European Journal of Applied Physiology, Berlin, v.108, n.5, p.945-55, 2010.

BELLINGER, B.M.; BOLD, A.; WILSON, G.R.; NOAKES. T.D.; MYBURGH, K.H. Oral creatine supplementation decreases plasma markers of adenine nucleotide degradation during a 1-h cycle test. Acta Physiologica Scandinavica, Stockholm, v.170, n.3, p.217-24, 2000.

BEMBEN, M.G.; LAMONT, H.S. Creatine supplementation and exercise performance: recent findings. Sports Medicine, Auckland, v.35, p.107-25, 2005.

BENDER, A.; AUER, D. P.; MERL, T.; REILMANN, R.; SAEMANN, P.; YASSOURIDIS, A.; BENDER, J.; WEINDL, A.; DOSE, M.; GASSER, T.; KLOPSTOCK, T. Creatine supplementation lowers brain glutamate levels in Huntington's disease. Journal of Neurology, Berlin, v.252, p.36-41, 2005.

BRANCACCIO, P.; MAFFULLI, N.; LIMONGELLI, F.M. Creatine kinase monitoring in sport medicine. British Medical Bulletin, London, v.81/82, n.1, p.209-30, 2007.

BRANNON, T.A.; ADAMS, G.R.; GONNIFF, C.L.; BALDWIN, K.M. Effects of creatine loading and training on running performance and biochemical properties of rat skeletal muscle. Medicine and Science in Sports and Exercise, Madison, v.29, p.489-95, 1997.

CHEUNG, K.; HUME, P.; MAXWELL, L. Delayed onset muscle soreness: treatment strategies and performance factors. Sports Medicine, Auckland, v.33, p.145-64, 2003.

CHEN, Y.; SERFASS, R.C.; APPLE, F.S. Alterations in the expression and activity of creatine kinase-M and mitochondrial creatine kinase subunits in skeletal muscle following prolonged intense exercise in rats. European Journal of Applied Physiology, Berlin, v.81, p.114-9, 2000.

COUDREUSE, J.M.; DUPONT, P.; NICOL, C. Delayed post effort muscle soreness. Annales de Réadaptation et de Médecine Physique, Amsterdam, v.47, p.290-8, 2004.

COOKE, M.B.; RYBALKA, E.; WILLIAMS, A.D.; CRIBB, P.J.; HAYES, A. Creatine supplementation enhances muscle force recovery after eccentrically-induced muscle damage in healthy individuals. Journal of the International Society of Sports Nutrition, Woodland Park, v.6, p.13, 2009.

DANGOTT, B.; SCHULTZ, E.; MOZDZIAK, P.E. Dietary creatine monohydrate supplementation increases satellite cell mitotic activity during compensatory hypertrophy. International Journal of Sports Medicine, Stuttgart, v.21, p.13-6, 2000. DEMINICE, R.; VILHENA, R.; PORTARI, G.V.; JORDÃO, A.A. Suplementação de creatina, homocisteína e estresse oxidativo. Medicina, Ribeirão Preto, v.40, n.3, p.368-77, 2007.

ENDOH, T.; NAKAJIMA, T.; SAKAMOTO, M.; KOMIYAMA, T. Effects of muscle damage induced by eccentric exercise on muscle fatigue. Medicine and Science in Sports and Exercise, Madison, v.37, p.1151-6, 2005.

GAGNON, M.; MAGUIRE, M.; MACDERMOTT, M.; BRADFORD, A. Effects of creatine loading and depletion on rat skeletal muscle contraction. Clinical Experimental Pharmacology and Physiology, Victoria, v.29, p.885-90, 2002.

GUALANO, B.; ARTIOLI, G.G.; POORTMANS, J.R.; LANCHA JUNIOR, A.H. Exploring the therapeutic role of creatine supplementation. Amino Acids, Wien, v.38, n.1, p.31-44, 2010.

GUYOTTO, L.C.C.; ALVES V.A.F. Métodos diagnósticos: provas de função hepática. In: GUYOTTO, L.C.C.; ALVES, V.A.F. Doenças do fígado e vias viliares. São Paulo: Atheneu, 2001. p.139-50.

HALLIWELL, B. Oxidative stress and neurodegeneration: where are we now? Journal of Neurochemistry, London, v.97, n.6, p.1634-58, 2006. 
KOMULAINEN, J.; TAKALA, T.E.; VIHKO, V. Does increased serum creatine kinase activity reflect exercise-induced muscle damage in rats? International Journal of Sports Medicine, Stuttgart, v.16, n.3, p.150-4, 1995.

KREIDER, R.B.; MELTON, C.; RASMUSSEN, C.J.; GREENWOOD, M.; LANCASTER, S.; CANTLER, E.C.; MILNOR, P.; ALMADA, A.L. Long-term creatine supplementation does not significantly affect clinical markers of health in athletes. Molecular and Cellular Biochemistry, Winnipeg, v.244, p.95-104, 2003.

LAVENDER, A.P.; NOSAKA, K. A light load eccentric exercise confers protection against a subsequent bout of more demanding eccentric exercise. Journal of Science and Medicine in Sport, Sydney, v.11, n.3, p.291-8, 2008.

LIEBER, R.L.; FRIDEN, J. Morphologic and mechanical basis of delayed-onset muscle soreness. Journal of the American Academy of Orthopaedic Surgeons, Rosemont, v.10, p.67-73, 2002.

MARGARITIS, I.; TESSIER, F.; VERDERA, F.; BERMON, S.; MARCONNET, P. Muscle enzyme release does not predict muscle function impairment after triathlon. Journal of Sports Medicine and Physical Fitness, Torino, v.39, n.2, p.133-9, 1999. MAYHEW, D.L.; MAYHEW, J.L.; WARE, J.S. Effects of long-term creatine supplementation on liver and kidney functions in American college football players. International Journal of Sport Nutrition and Exercise Metabolism, Tallahassee, v.12, p.453-60, 2002.

MENEZES, L.G.; SOBREIRA, C.; NEDER, L.; RODRIGUES-JÚNIOR, A.L.; MARTINEZ, J.A. Creatine supplementation attenuates corticosteroid-induced muscle wasting and impairment of exercise performance in rats. Journal of Applied Physiology, Bethesda, v.102, n.2, p.698-703, 2007.

NAKAO, C.; OOKAWARA, T.; KIZAKI, T.; OH-ISHI, S.; MIYAZAKI, H.; HAGA, S.; SATO, Y.; JI, L.L.; OHNO, H. Effects of swimming training on three superoxide dismutase isoenzymes in mouse tissue. Journal of Applied Physiology, Bethesda, v.88 p.649-54, 2000.

NEUBAUER, O.; KONIG, D.; WAGNER, K.H. Recovery after an Ironman triathlon: sustained inflammatory responses and muscular stress. European Journal of Applied Physiology, Berlin, v.104, n.3, p.417-26, 2008.

NOSAKA, K.; NEWTON, M.; SACCO, P.; CHAPMAN, D.; LAVENDER, A. Partial protection against muscle damage by eccentric actions at short muscle lengths. Medicine and Science in Sports and Exercise, Madison, v. 37, n. 5, p.746-53, 2005. OSORIO, R.A.; CHRISTOFANI, J.S.; D’ALMEIDA, V.; RUSSO, A.K.; PICARRO, I.C. Reactive oxygen species in pregnant rats: effects of exercise and thermal stress. Comparative Biochemistry and Physiology: Part C Toxicology \& Pharmacology, New York, v.135, p.89-95, 2003a.

OSORIO, R.A.; SILVEIRA, V.L.; MALDJIAN, S.; MORALES, A.; CHRISTOFANI, J.S.; RUSSO, A.K. Swimming of pregnant rats at different water temperatures. Comparative Biochemistry and Physiology: Part A, Molecular \& Integrative Physiology, New York, v.135, p.605-11, 2003b.

PERSKY, A.M.; BRAZEAU, G.A. Clinical pharmacology of the dietary supplement creatine monohydrate. Pharmacological Reviews, Baltimore, v.53, n.2, p.161-76, 2001.

RAWSON, E.S.; CONTI, M.P.; MILES, M.P. Creatine supplementation does not reduce muscle damage or enhance recovery from resistance exercise. Journal of Strength and Conditioning Research, Champaign, v.21, n.4, p.1208-13, 2007. RAWSON, E.S.; GUNN, B.; CLARKSON, P.M. The effects of creatine supplementation on exercise-induced muscle damage. Journal of Strength and Conditioning Research, Champaign, v.15, p.178-84, 2001.

SAKS, V.A.; STRUMIA, E. Phosphocreatine: molecular and cellular aspects of the mechanism of cardioprotective action. Current Therapeutic Research, Clinical and Sxperimental, New York, v.53, p.565-98, 1993.

SANTOS, R.V.; BASSIT, R.A.; CAPERUTO, E.C.; COSTA ROSA, L.F. The effect of creatine supplementation upon inflammatory and muscle soreness markers after a $30 \mathrm{~km}$ race. Life Sciences, Oxford, v.75, p.1917-24, 2004.

SESTILI, P.; MARTINELLI, C.; BRAVI, G.; PICCOLI, G.; CURCI, R.; BATTISTELLI, M.; FALCIERI, E.; AGOSTINI, D.; GIOACCHINI, A.M.; STOCCHI, V. Creatine supplementation affords cytoprotection in oxidatively injured cultured mammalian cells via direct antioxidantactivity. Free Radical Biology \& Medicine, New York, v.40, n.5, p.837-49, 2006.

SOUZA, R.A.; MIRANDA, H.; XAVIER, M.; OSORIO, R.A.; GOUVEA, H.; COGO, J.C.; VIEIRA, R.P.; RIBEIRO, W. Effects of high-dose creatine supplementation on kidney and liver responses in sedentary and exercised rats. Journal of Sports Science and Medicine, Ankara, v. 8, p.672-81, 2009.

SOUZA, R.A.; SANTOS, R.M.; OSÓRIO, R.A.; COGO, J.C.; PRIANTI JÚNIOR, A.C.G.; MARTINS, R.A.B.L.; RIBEIRO, W. Influência da suplementação aguda e crônica de creatina sobre as concentrações sanguíneas de glicose e lactato de ratos Wistar. Revista Brasileira de Medicina do Esporte, Rio de Janeiro, v.12, n.6, p.361-5, 2006.

TARNOPOLSKY, M.A.; BOURGEOIS, J.M.; SNOW, R.; KEYS, S.; ROY, B.D.; KWIEEIEN, J.M, TURNBULL, J. Histological assessment of intermediate and long-term creatine monohydrate supplementation in mice and rats. American Journal of Physiology: Regulatory, Integrative and Comparative Physiology, Bethesda, v.285, p.762-9, 2003. 
TERJUNG, R.L.; CLARKSON, P.; EICHNER, E.R.; GREENHAFF, P.L.; HESPEL, P.J.; ISRAEL, R.G.; KRAEMER, W.J.; MEYER, R.A.; SPRIET, L.L.; TARNOPOLSKY, M.A.; WAGENMAKERS, A.J.; WILLIAMS, M.H. American College of Sports Medicine roundtable. The physiological and health effects of oral creatine supplementation. Medicine and Science in Sports and Exercise, Madison, v. 32, p.706-17, 2000.

VOLTARELLI, F.A.; GOBATTO, C.A.; MELLO, M.A.R. Determination of anerobic threshold in rats using the lactate minimum test. Brazilian Journal of Medical and Biological Research, Ribeirão Preto, v.35, p.1389-94, 2002.

WARREN, G.L.; FENNESSY, J.M.; MILLARD-STAFFORD, M.L. Strength loss after eccentric contractions is unaffected by creatine supplementation. Journal of Applied Physiology, Bethesda, v.89, p.557-62, 2000.

WYSS, M.; SCHULZE, A. Health implications of creatine: can oral creatine supplementation protect against neurological and atherosclerotic disease? Neuroscience, Oxford, v.112, n.2, p.243-60, 2002.

YOUNG, R.E.; YOUNG, J.C. The effect of creatine supplementation on mass and performance of rat skeletal muscle. Life Sciences, Oxford, v. 81, p.710-16, 2007.

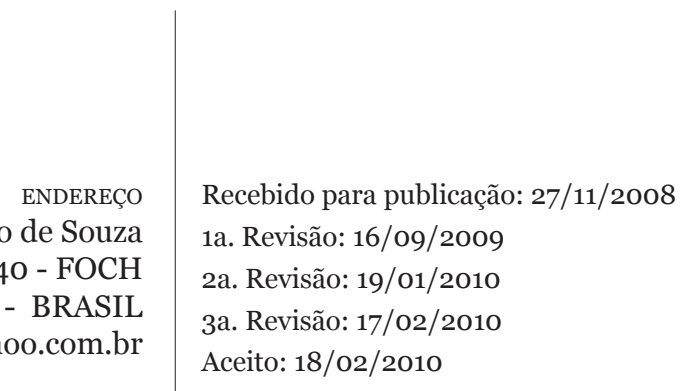

R. João Nunes de Oliveira Junior, 140 - FOCH 37550-0oo - Pouso Alegre - MG - BRASIL e-mail: tatosouza2004@yahoo.com.br Aceito: 18/02/2010 